\title{
An Air-ground Robotic Cooperative System for Explosive Disposal
}

\author{
Hanqing Tian ${ }^{1,}{ }^{*}$, Jiahe Li, Tianfang Liu and Guangming Xiong \\ ${ }^{1} 5$ South Zhongguancun Street, Haidian District, Beijing,China \\ afanmeiling68@126.com
}

\begin{abstract}
Keywords: air-ground cooperation; environment detecting; exploder disposal
Abstract : An air-ground cooperative system is proposed for exploder detecting and disposal aiming at unknown and hazardous environments such as explosive disasters. The ground robot as the mobile platform, and the unmanned air vehicle follows the ground robot for real-time environment detecting using its superiority in air view. The computer shows the video all the time and the professor should identify it when the potential exploder is detected. If it is determined as dangerous goods, the robot travels to the site and crawl through the front of the robot manipulator of dangerous goods to the designated treatment area, making secure detonation or other appropriate treatment.
\end{abstract}

\section{Introduction}

The collaborative control system makes the ground robot as the mobile and host platform in charge of multiple tasks such as the UAV take-off, the arm installation and the transfer of dangerous materials. Since the application environment is complex and unknown, the ground robot need to facilitate handling, flexible, have a smaller turning radius and higher ground mobility, and can ensure adequate range.

Compared with ground robots, the UAV is the ideal environment detection tool because it has a broader view from the sky, which can provide a larger range of the view. It is flexible and easy to manipulate. However, because of the limitation of loading and other aspects, it need collaborate with the ground robot [1].

The main task of the closed-loop control system can be summarized as: the UAV real-time adjusts its posture and detects the surrounding environment following the ground mobile robot: Image transferred from UAV will get to the computer. One aspect, for image processing, PID control algorithm processing and generating control orders feeding back to the drone in order to auto-follow the ground robot by visual method, another for displaying on the screen for the operator to do observation and identification: the operator remotely controls the robot motion, grabbing dangerous objects by mechanical arm and transporting it to the designated place to dispose accordingly based on the image information; the UAV will move based on the motion of the ground robot in the same time.

According to the task description, the project can be roughly divided into four functional modules:

(1) The module of computer A is responsible for computer image processing, PID control algorithm and the image display;

(2) The computer B module includes instructions to send robots and manipulators;

(3) The UAV module is for independent following, flight control and environmental detection;

(4) The ground mobile robot platform module includes p3-at control, the manipulator control, identification and handling of dangerous goods.

\section{Hardware}

The platform of the image acquisition device uses the French Parrot company product---four-axis remote control helicopter AR.Drone 2.0, which has two cameras, one in front (1280*720 pixels), and another is below (toward the ground, $320^{*} 240$ pixels). In this paper, the below camera will be used to obtain an image.

In this study the wheeled mobile robot is the Pioneer3-AT, which is shown in Fig. 2 [2] 
Other equipment include: a wireless transmission module ZigBee, model SSET RS232 and the dangerous goods manipulator gripping device whose structure is separately designed.

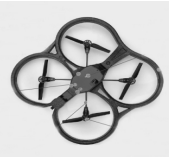

Fig 1. AR.Drone 2.0

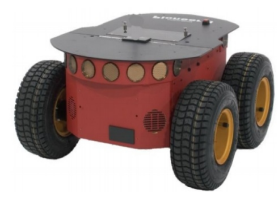

Fig. 2. P3-AT ground robot

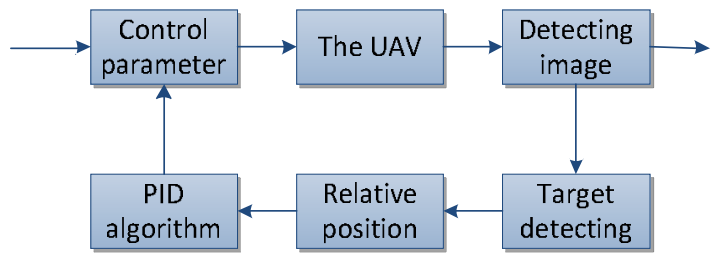

Fig. 3. Image processing chart 1

\section{Target following based on image processing}

The relative position and speed between the target pattern and the drone is needed in order to control the UAV to trace the pattern. Therefore, we need target detection, the relative position acquisition, PID control algorithm and other treatment. Relevant processes are shown in Fig.3.

\section{Target detection}

The image of environment detection should include the environmental information and the information of the following target. The main purpose of image processing is to exclude the interference of other objects detected green circle labeled samples and calculate the centroid position as the target position following control.

Target detection needs to call image frame, extract the part of the green point as the following target and connected domains meeting the requirements will be marked the centroid position. Consider the lighting conditions and other environmental factors, we also need to analyze specific information about $\mathrm{R}$ and $\mathrm{B}$ channels in the image when we identify green information [3].

The principle is as follows: firstly, make the image binarization processing. The specific process is to acquire the image of each pixel R, G, B channel data and calculate the difference value with other two channels of data, if the difference value is greater than a predetermined threshold value, then the pixel is set to 1 , and 0 otherwise; Secondly, sort and filter the connected components by bubble algorithm to extract the maximum communication domain meeting the requirements, which is the mark dot; Finally, obtain the centroid of the maximize connected domain.

\section{Get the relative position}

Since the position of UAVsuch as pitch angle and yaw angle are in target following, the camera cannot be perpendicular to the ground in real time, so it cannot determine the relative position of the UAV and the target object only by the image information and height data. Therefore, it must obtain an attitude angle and height information, establish the angle picture frame model, to determine the relative position of the drone and the ground robot in the Earth coordinate system through coordinate conversion.

From the above chart we can see that there is an angular relationship between the earth coordinate system and the certain image frame coordinate. We should firstly do spatial coordinate system conversion in calculating the relative position. In the spatial coordinate system conversion, we consider to have the same origin of coordinates, so it will be completed after three rotations, which is called Euler angle rotating $\varepsilon_{X}, \varepsilon_{Y}, z_{Z}$ around axis OX, OY and OZ [4].

The coordinate transformation matrix as follows :

$$
=\left[\begin{array}{ccc}
\mathbb{R}(\varepsilon)=R_{1}\left(\varepsilon_{X}\right) R_{2}\left(\varepsilon_{y}\right) R_{Z}\left(\varepsilon_{Z}\right) \\
\cos \varepsilon_{X} \sin \varepsilon_{Z}+\sin \varepsilon_{X} \sin \varepsilon_{Y} \cos \varepsilon_{Z} & \cos \varepsilon_{X} \cos \varepsilon_{Z}+\sin \varepsilon_{X} \sin \varepsilon_{Y} \sin \varepsilon_{Z} & -\sin \varepsilon_{X} \cos \varepsilon_{Y} \\
-\cos \varepsilon_{Y} \cos \varepsilon_{Z} & \cos \varepsilon_{Y} \sin \varepsilon_{Z} & \sin \varepsilon_{Y} \\
\sin \varepsilon_{X} \sin \varepsilon_{Z}+\cos \varepsilon_{X} \sin \varepsilon_{Y} \sin \varepsilon_{Z} & -\sin \varepsilon_{X} \cos \varepsilon_{Z}+\cos \varepsilon_{X} \sin \varepsilon_{Y} \sin \varepsilon_{Z} & \cos \varepsilon_{X} \cos \varepsilon_{Y}
\end{array}\right]+
$$


Therefore, the spatial coordinate conversion formula is that :

$$
\left[\begin{array}{l}
X \\
Y \\
Z
\end{array}\right]=\mathrm{R}(\sigma)\left[\begin{array}{c}
Y_{0} \\
Y_{0} \\
Z_{0}
\end{array}\right] \text {. }
$$

Where, $\left[\begin{array}{lll}x & y & z\end{array}\right]^{T}$ is the target coordinate ;

$\left[\begin{array}{lll}X_{0} & Y_{0} & Z_{0}\end{array}\right]^{T}$ is the original coordinate。

Formula (2) can convert the image coordinate to earth coordinate system and then get the actual relative positions.

PID algorithm

Target following algorithm use the traditional PID control algorithm, using the speed deviation as the control variable. After adjusting, only parts of the algorithm P and D are used to achieve the goal that following the target. Wherein, $\mathrm{P}$ is used to control the UAV to the target position, $\mathrm{D}$ is used to reduce the overshoot. The process as follows: After obtaining the relative position of the UAV and the labeled sample, you can get the next time target speed of the UAV by controlling the amount of time, combined with their current speed to calculate the current speed deviation through PID algorithm and output UAV pitch angle and yaw angle for the UAV control . Control flow chart is shown in Fig.4.

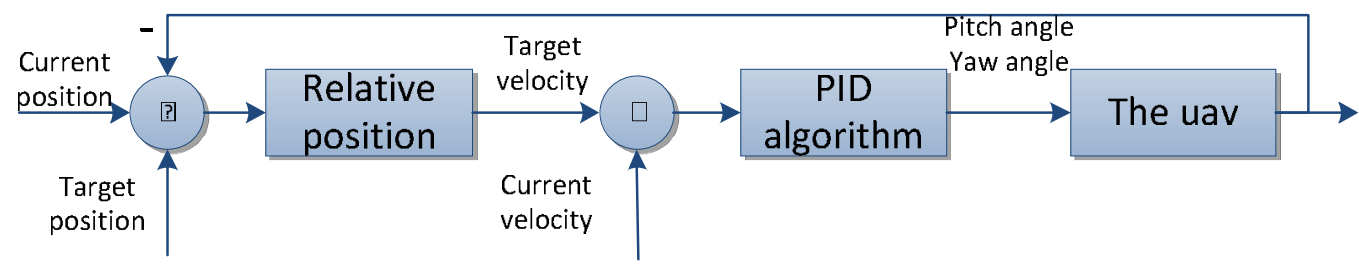

Fig. 4. Control flow

\section{Ground robot control based on wireless transmission}

ZigBee wireless communication networks supports star network topology, tree topology, mesh network topology, chain topology and network topologies peer topology. And in data of transmission modes, it includes broadcast mode, master-slave mode, point to point mode[6][7].

The final form of free network is shown in Fig.5.

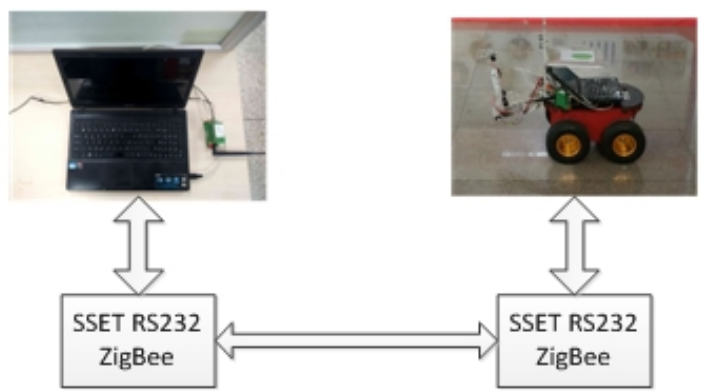

Fig.5. The form of free network

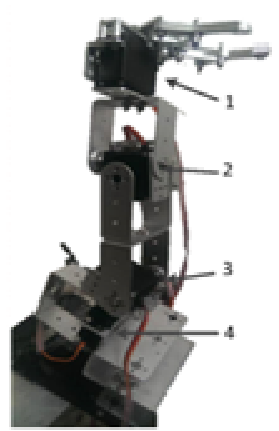

Fig.6. The manipulator

\section{Design and control of multi-DOF manipulator}

The manipulator remote control includes 8-channel transmitter and its transmission frequency band is $2.4 \mathrm{GHz}$, which can output 8 -channel PWM wave corresponding to the data receiver. The receiver may provide a signal directly to the joint servo control [8]. The final design is shown in Fig.6. 
Four(1,2,3 and 4) servos are numbered from top to bottom, providing a total of three degrees of freedom movement. The first servo controls the opening and closing mechanical clamps for gripping stuff; the second servo controls turning left and right front-end for adjusting the most distal; the third steering gear is lifting node to lift up and put down the object by using the robot arm pitch; the fourth controls a wide range of motion by rotating around the control arm.

\section{Experimental Verification}

The test results verify three main aspects: First, whether the ground robot control is accurate by wireless transmission; second, whether the UAV can autonomously follow the ground and provide a clear picture; third, whether the design and control of arm is able to meet the crawl requirements.

During the test we found that the ground mobile robots via wireless transmission module can be accurately controlled by computer. The UAV can maintain in following and provide enough stable images. The operator can manipulate the robotic arm to complete the article crawling task. Test procedure is shown in Fig.7.

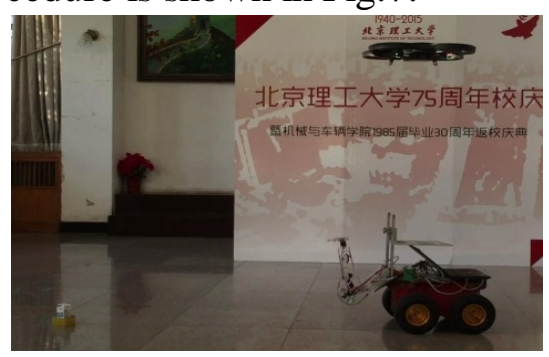

(a) The UAV follow

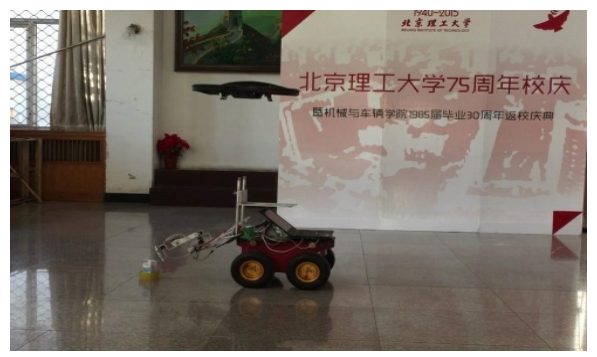

(b) Grab the object

\section{References}

[1] Lee, MFR, Li, YC, Chien, MY . Real-time face tracking and recognition using the mobile robots[J]. ADVANCED ROBOTICS,2015.187-208

[2] a-Ruey Chang; Feng-Chi Yu; Tsun-Cheng Huang; Shih-Chung Kang; Shang-Hsien Hsieh. The Use of a VRS-Based Pioneer 3-A Robot in Pavement Inspections [C]. Journal of Testing and Evaluation.2011, 9

[3] akahara T , Yamashita Y ,et. "GPT Correlation for 2D Projection Transformation Invariant Template Matching”. Pattern Recognition (ICPR), 2014 22nd International Conserence:3810-3815

[4] Yong, L. A correspondence finding method based on space conversion in 3D shape measurement using fringe projection[J]. OPTICS EXPRESS.2015,6; 14188-14202.

[5] Yinsong Wang, Hongliang Zheng. A New PID Control Method for Hydraulic Turbine Governor[C].Applied Mechanics and Materials.2013

[6] Badescu, AM,Cotofana, L . A wireless sensor network to monitor and protect tigers in the wild[J]. ECOLOGICAL INDICATORS.2015,5;447-457

[7] Y. Li, G. M. Xiong, P. Liu, Y. J. Sun, J. W. Gong. Design of a Multi-P3-AT-Robot Platform Using Ad Hoc Network [C]. Advanced Materials Research, 2013, 7: 2208-2213.

[8] Islam, S , Liu, PX , El Saddik, A . Nonlinear adaptive control for teleoperation systems with symmetrical and unsymmetrical time-varying delay[J]. INTERNATIONAL JOURNAL OF SYSTEMS SCIENCE,2015.2928-2938 